\title{
HIDROGEOLOGÍA DE OJO DE AGUA, CUENCA SUR DE LA CIUDAD DE GUATEMALA
}

\author{
HIDROGEOLOGY OF OJO DE AGUA, SOUTH BASIN OF GUATEMALA CITY
}

\author{
Isaac R. Herrera* \& Eugenio O. Orozco \\ Universidad de San Carlos de Guatemala, Facultad de Agronomía \\ Código Postal USAC 01012. Ciudad Universitaria Zona 12, Guatemala \\ *Autor para contacto: iherrerai@hotmail.com
}

(Recibido: 02/11/2007; aceptado: 22/06/2010)

\begin{abstract}
RESUMEN: En este trabajo se presentan las características geológicas e hidrogeológicas del sector de Ojo de Agua en la cuenca sur de la ciudad de Guatemala, donde se ubica un campo de pozos que produce un $72 \%$ del caudal extraído de los acuíferos del Valle Central y un poco más del $20 \%$ de la producción total para la ciudad de Guatemala. Este sector es de vital importancia para el abastecimiento de agua potable, planteándose la necesidad de seguir aprovechando este acuífero en forma sostenible, haciéndose necesaria esta investigación para determinar el modelo hidrogeológico conceptual y establecer nuevos sitios de perforación de pozos. La geología del área incluye lavas, sedimentos y piroclastos de permeabilidad variable, dando origen a dos acuíferos, uno superior y otro inferior. El superior está constituido por material de relleno de piroclastos y sedimentos fluviales, con permeabilidad primaria, de tipo libre, con valores de transmisividad para los piroclastos de 50 a $750 \mathrm{~m}^{2} /$ día, y de 150 a $2000 \mathrm{~m}^{2} /$ día para los sedimentos. Los valores de coeficiente de almacenamiento de los piroclastos es 0,09 y para los depósitos aluviales de 0,20. El acuífero inferior presenta una permeabilidad secundaria, encontrándose también bajo condiciones de confinamiento. La transmisividad oscila entre 1600 y $9500 \mathrm{~m}^{2} /$ día en los pozos de Ojo de Agua, con un valor de coeficiente de almacenamiento para rocas volcánicas fracturadas de 0,014. Los niveles estáticos de los pozos de Ojo de Agua han descendido hasta 13,2 m con un promedio de $9 \mathrm{~m}$ en más de 20 años. Actualmente, la profundidad del nivel de agua subterránea con respecto al nivel del suelo varía de 12 y 14 m, existiendo anteriormente manantiales surgentes. El caudal de los principales pozos varía de 134 a 232 l/s, con un caudal total de 1675 l/s. La dirección de flujo de las aguas subterráneas es principalmente de norte a sur y tiene un control tectónico por un sistema de fallas con orientación N-S, que ha originado estructuras de horsts y grábenes en las cercanías.

Palabras clave: Acuífero, Ojo de Agua, permeabilidad, transmisividad, ciudad de Guatemala y graben.
\end{abstract}

\begin{abstract}
This paper presents the geologic and hydrogeologic features of the Ojo de Agua zone in the southern basin of Guatemala City where a battery of water wells produces around $72 \%$ of the groundwater extracted from the aquifers of the central valley. The exploited outflow at Ojo de Agua represents $20 \%$ of the total production of Guatemala City. Therefore, Ojo de Agua is of essential importance for the water supply of the city making it is necessary to be exploit in a sustainable manner. This research is necessary to determine a conceptual hydrologic model and to establish new sites for water wells. The geology is conformed by lava flows, pyroclasts and sediments of variable permeability.
\end{abstract}


An upper aquifer and lower aquifer have been established. The upper aquifer is conformed by pyroclasts and lake and fluvial sediments, with primary permeability and unconfined type. The aquifer has transmisivity values of 50 to 750 $\mathrm{m}^{2} /$ day for the pyroclastic materials, and 150 to $2000 \mathrm{~m}^{2} /$ day for the sediment materials. The storage coefficient is 0,09 and 0.20 respectively. The lower aquifer is confined and has secondary permeability; transmisivity varies from 1600 to $9500 \mathrm{~m}^{2} /$ day and the volcanic rocks have a storage coefficient of 0,014 . The static levels of the water wells of Ojo de Agua have been fell down an average of $9 \mathrm{~m}$ in a period of over 20 years; the maximum drop registered is $13,2 \mathrm{~m}$. The actual groundwater level depth is 12 to $14 \mathrm{~m}$ below ground level. Surging springs were reported in the area before the groundwater exploitation. The outflow of the main water wells varies from 134 to $232 \mathrm{l} / \mathrm{s}$, for a total of 1675 1/s. The direction of groundwater flow is mainly north-south, controlled by a fault system with the same orientation that has developed horst and graben structures nearby.

Key words: Aquifer, Ojo de Agua, permeability, transmisivity, Guatemala city, graben.

\section{INTRODUCCIÓN}

Durante las últimas tres décadas, la ciudad de Guatemala ha crecido alarmantemente hasta tener más de dos millones de habitantes (Instituto Nacional de Estadística, 2002). Paralelamente a esto ha sucedido un crecimiento industrial, dando como resultado altos niveles de contaminantes en las aguas superficiales que las hace inadecuadas para el consumo humano, dándose el incremento en la explotación de las aguas subterráneas (Herrera, 2002), ya que estos recursos son los más factibles de explotar en forma inmediata para satisfacer las necesidades de agua potable de la ciudad.

La ciudad de Guatemala actualmente se abastece de agua por medio de fuentes subterráneas y aguas superficiales. Las aguas subterráneas representan más del $40 \%$ del abastecimiento de agua potable actual, entregado por la Empresa Municipal de Agua (EMPAGUA). El sistema de pozos del sector Ojo de Agua y Diamante producen un 72\% del caudal extraído de los acuíferos y un poco más del 20\% de la producción total (Velásquez, 1998). La fuente del sector de Ojo de Agua es de vital importancia para el abastecimiento a largo plazo y se plantea la necesidad de seguir explotando este acuífero en forma técnica, haciéndose necesaria esta investigación para establecer nuevos sitios de perforación de pozos.

El objetivo de este trabajo es determinar las características geológicas e hidrogeológicas del sector de Ojo de Agua, para definir la hidroestratigrafía volcánica de las áreas con alta permeabilidad y con un buen flujo de agua subterránea, para recomendar posibles puntos de perforación de pozos en los alrededores del sector. Se definió un cuadrante de $24 \mathrm{~km}^{2}$ que se encuentra entre las coordenadas geográficas $14^{\circ} 30,87^{\prime}$ a $14^{\circ} 34,13^{\prime}$ $\mathrm{N}$ y $90^{\circ} 31,93^{\prime}$ a $90^{\circ} 34,17^{\prime} \mathrm{O}$ (Fig. 1).

El área comprende desde Ciudad Real a Villa Nueva a aproximadamente $13 \mathrm{~km}$ al sur del centro de la ciudad de Guatemala. La ciudad de Guatemala está atravesada por la divisoria continental de aguas superficiales, formando las cuencas del río Las Vacas al norte y la cuenca del río Villalobos al sur (donde se encuentra la zona de interés). El área de estudio se encuentra limitada por el río Villalobos al oeste y el río Pinula al este, corriendo ambos en sentido norte a sur (INSIVUMEH-IGN-ONU, 1978). Las elevaciones son de 1250 a más de 1400 m s.n.m., siendo el lugar más alto el Cerro Gordo (1440 m s.n.m.) al centro del área y el más bajo el pequeño delta del río Villalobos al sur. De tal cuenta, la pendiente se orienta de norte a sur y varía de 8 a $14 \%$ en las partes altas y de 1 a $4 \%$ en las partes bajas.

\section{GEOLOGÍA}

Para investigar la geología del sector y la distribución de las rocas en profundidad se utilizaron tres fuentes de información: pozos (López, 1989; Jiménez, 1990; EMPAGUA, 1996), geología (Koch \& McLean, 1977) e hidrogeología de campo (GEOCONSA, 1999). En primera instancia se ha utilizado información de 14 perforaciones, que se complementó con la geología de campo obtenida por medio de observación de afloramientos en los ríos Villalobos y Pinula principalmente.

La geología del área de estudio se presenta en el mapa geológico (Fig. 2), en el cual se incluye en 
forma general a las unidades de interés hidrogeológico. El basamento está formado por flujos de lavas andesíticas del Terciario (Koch \& McLean, 1977), que constituye en gran porcentaje la zona saturada, mientras que la zona no saturada está constituida principalmente por piroclastos del Cuaternario, como se muestra en los perfiles geológicos de las figuras 3 y 4 . Como se observa en el perfil geológico A - A', en la parte sur del área del río Villalobos, los materiales sedimentarios están constituidos por sedimentos fluvio-lacustres con tefras y diamictones pumíticos, así como los aluviones del río. De manera parecida, en el perfil geológico B - B', se observa que las partes este y oeste de los ríos Pinula y Villalobos respectivamente, se presentan los sedimentos recientes.

\section{Estratigrafía}

La estratigrafía de los alrededores del sector de Ojo de Agua se presenta en la Figura 5, donde se observan las lavas andesíticas del Terciario, los depósitos fluvio-lacustres con tefra y diamictones pumíticos cuaternarios, las tefras o piroclastos del Cuaternario y aluviones (Koch \& McLean, 1977).

\section{Lavas andesiticas del Terciario}

Unidad constituida por flujos de lavas de composición andesítica, formando el basamento sobre el cual se depositaron los depósitos cuaternarios originados en la formación de la caldera del lago de Amatitlán, así como depósitos volcánicos originados por las erupciones de otros volcanes principalmente de Agua y Atitlán y recientemente del volcán de Pacaya (Herrera, 2002).

En el área de estudio, los afloramientos son escasos, identificándose solo tres afloramientos. El primero en el área de Ojo de Agua, donde la andesita se encuentra parcialmente alterada y es de color pardo grisáceo a pardo violáceo, compuesta por fenocristales blancos de plagioclasa, algunos oscuros de piroxenos y biotita, y amarillentos de limonita. La textura es traquítica a fluidal, con algunas vacuolas pequeñas. La roca está diaclasada verticalmente $\left(70^{\circ}\right.$ a $\left.90^{\circ}\right)$ en sentido N-S y

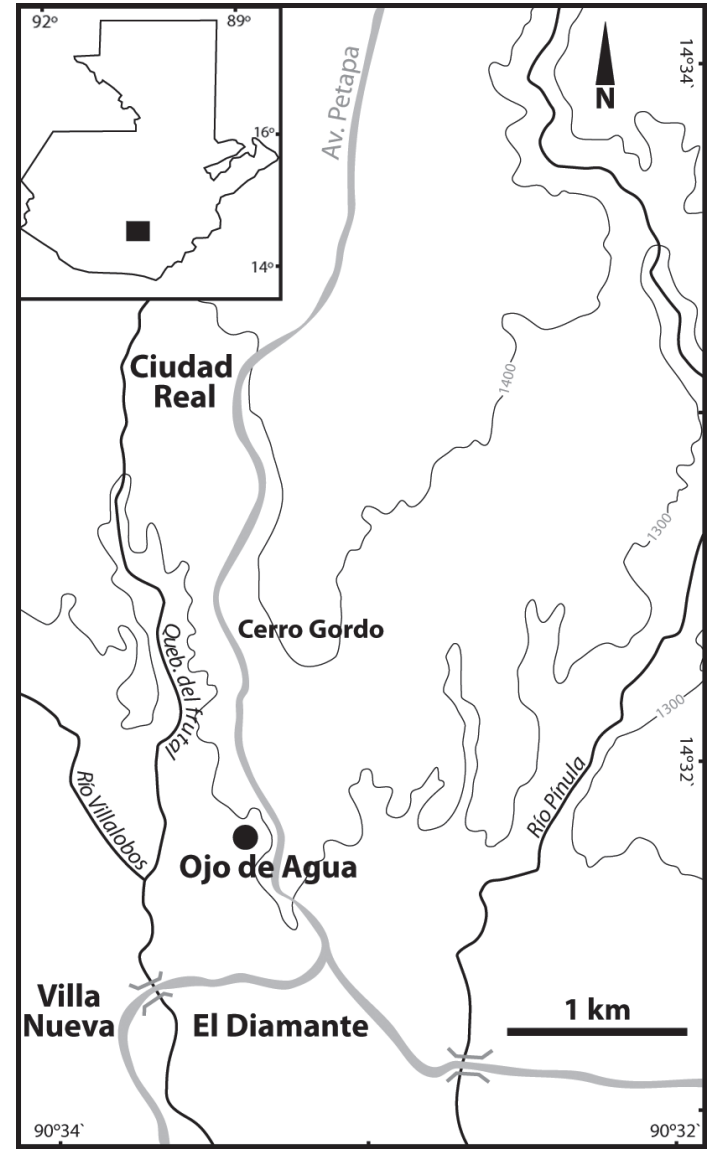

Fig. 1: Mapa de ubicación

otras en sentido E-W, siendo las diaclasas abiertas y centimétricas (5 a $40 \mathrm{~cm}$ ), por lo que la roca se presenta en forma de bloques centimétricos a métricos.

El segundo afloramiento se identifica al sur del cerro Gordo, donde las lavas andesíticas son de color gris claro, compuesta por fenocristales de plagioclasa, piroxenos, biotita y óxidos de hierro. En algunas partes se observan lavas dacíticas de apariencia fluidal y se encuentran en bloques basculados con seudobuzamiento de $5^{\circ}$ al N85 $\mathrm{W}$. Un tercer afloramiento se presenta en la quebrada del Frutal al oeste de Ciudad Real como se observa en el mapa geológico. La importancia que tiene esta unidad de lavas, desde el punto de vista hidrogeológico, es que se encuentran muy diaclasadas y es a través de las fracturas por donde circula el agua del acuífero principal de la región sur del 
valle de Guatemala. El espesor de esta unidad no se conoce con certeza, pero se supone mayor de 300 metros (Velásquez, 1998).

\section{Depósitos fluvio-lacustres con tefra y diamictones pumiceos Cuaternarios}

Esta unidad corresponde a una secuencia de intercalaciones de depósitos fluvio-lacustres finos interestratificados con los diamictones pomíceos. Los sedimentos lacustres son de diatomitas formadas en un ambiente del paleolago de Amatitlán. Además se presentan depósitos fluviales, que corresponden a paleocauces que fueron afluentes del paleolago, que dieron como resultado la depositación de arenas de color verde y gris, con abundantes granos de cuarzo, biotita, pómez $\mathrm{y}$ andesita, que se presentan de forma laminada entre 10 a $30 \mathrm{~cm}$ de espesor.

El término diamictón se refiere principalmente a los flujos volcánicos no clasificados que rellenaron valles, introducido por Koch \& McLean (1975), denominado como "Flujo los Chocoyos", que se caracteriza por presentar una zona de color rosado ("pink zone"). Esta unidad se localiza principalmente en la parte central y sur del área, cubriendo principalmente las partes planas en donde se originó la deposición de estos materiales finos de forma alterna, presentándose pequeños estratos de tierra de diatomea, de color blancuzco, muy porosa y poco densa, con espesores de 0,4 a $0,8 \mathrm{~m}$ y con buzamientos horizontales y algunos ligeramente inclinados entre $3^{\circ}$ a $5^{\circ}$ al sur. Los depósitos piroclásticos corresponden con granos de pómez de tamaño lapilli, de color gris a blanco amarillento, angulares y subangulares y con capas de 0,15 a $0,2 \mathrm{~m}$ de espesor.

\section{Tefras o piroclastos del Cuaternario}

Se localizan en la parte norte del área, siendo materiales de color pardo amarillento, compuestos de bloques de andesitas grisáceos entre 5 a 45

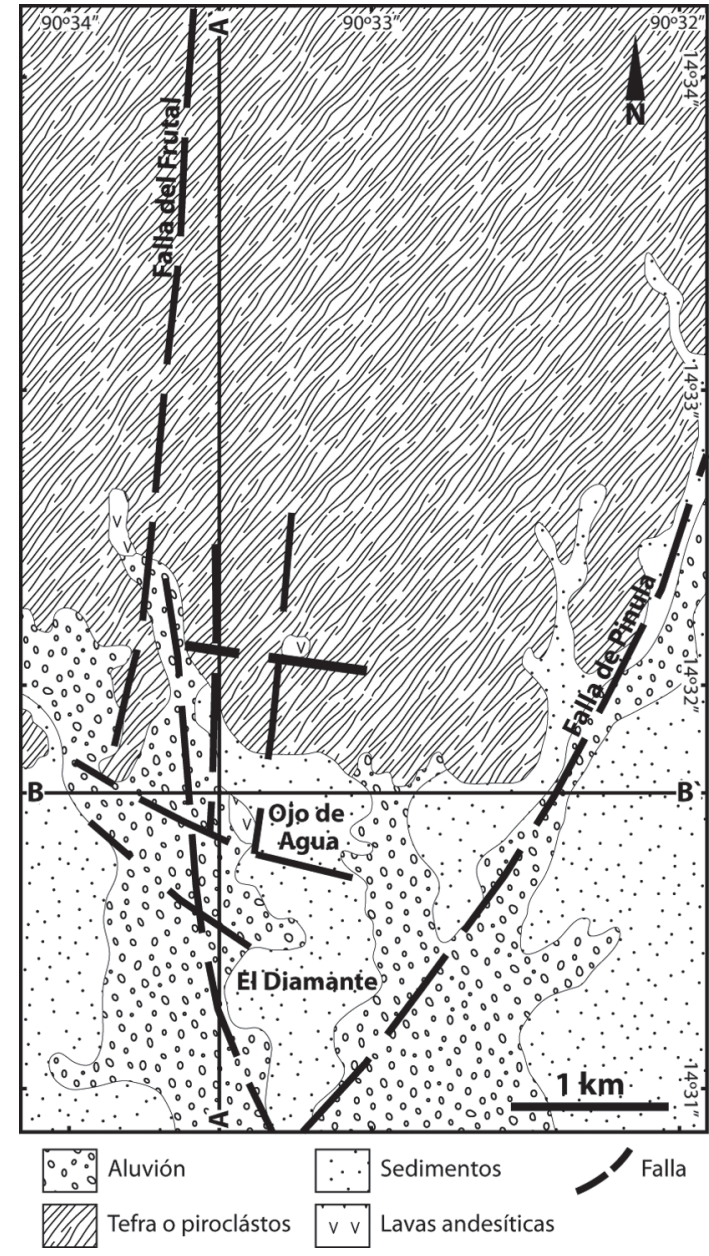

Fig. 2: Mapa geológico

cm de tamaño, de forma angular y subredondeada. La matriz está compuesta por ceniza, lapilli, escoria y pómez blanca con mucho vidrio volcánico, cuarzo, minerales máficos y fragmentos líticos.

La unidad de tefra es de mucha importancia porque constituye en gran porcentaje la zona no saturada dentro del valle de Guatemala. El informe de BRGM-INSIVUMEH (1997) menciona que esta unidad de piroclastos es importante como capa geopurificadora, que funciona como un filtro natural para evitar el transporte hacia la zona saturada de algunos contaminantes biológicos y de ciertos metales. 


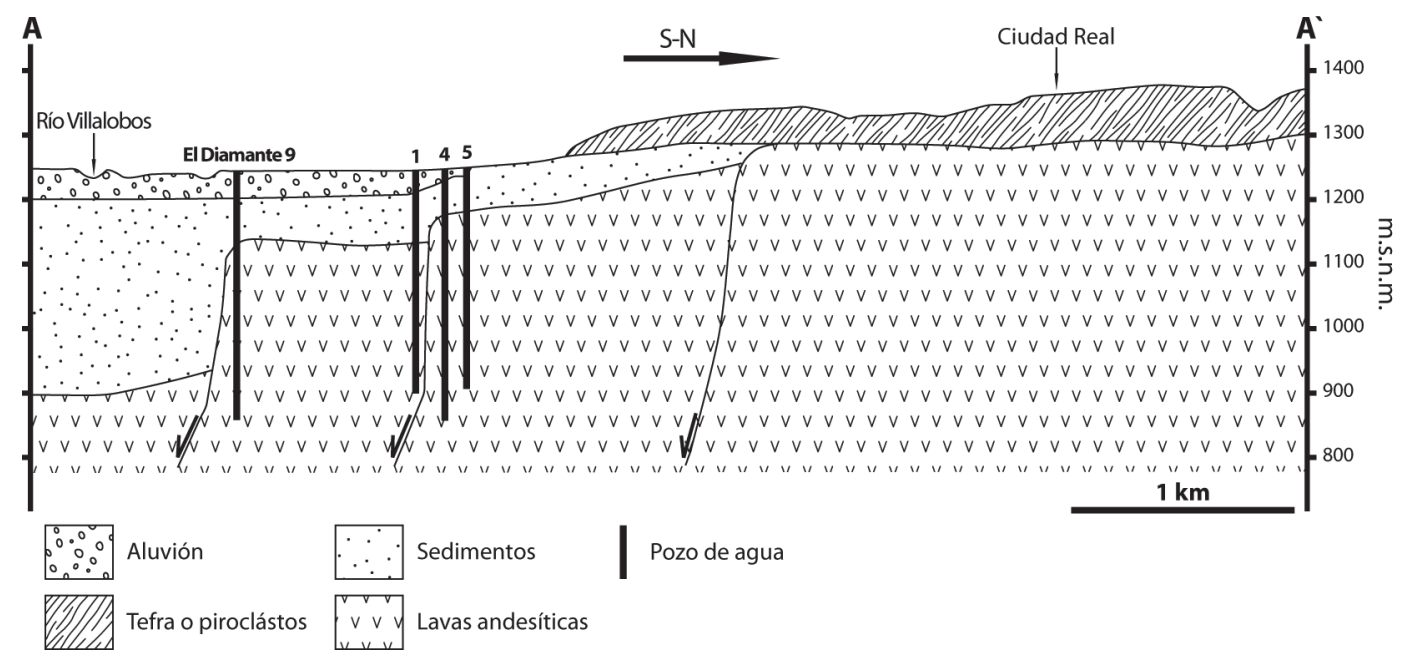

Fig. 3: Perfil geológico A-A'

\section{Depósitos aluviales del Cuaternario}

Estos depósitos yacen principalmente a lo largo de los valles y de las riberas de los ríos Villalobos y Pinula. La unidad se compone de sedimentos secundarios de los materiales volcánicos. Es una unidad heterogénea con sedimentos finos de pómez y ceniza volcánica, que alternan con sedimentos gruesos (cantos rodados) de piedra pómez, dacitas, riodacitas y andesitas, con espesores variables, generalmente de 10 a $30 \mathrm{~m}$.

\section{Geología estructural}

La ciudad de Guatemala se encuentra en un valle tectónico que corresponde a un graben (depresión tectónica) limitado por horsts (pilares tectónicos) (GEOCONSA, 1999). Dentro de la fisiografía del Valle de Guatemala es muy evidente el control estructural dado por los sistemas de fallas de Mixco y Santa Catarina Pinula, las cuales son fallas normales y de orientación $\mathrm{N}-\mathrm{S}$, fácilmente identificables por su escarpe de falla y que definen la estructura de graben de la ciudad de Guatemala.
Los flujos de lava se encuentran parcialmente tectonizados (que en algunos casos dan la apariencia de una pizarra), resultado del levantamiento de la región, y específicamente por movimientos del sistema de fallas de Mixco al oeste del valle. Esto es importante ya que las rocas están altamente fracturadas y es por donde circula el agua subterránea, llegando a constituir el acuífero principal de la región, delimitado por sistemas de fallas paralelas con una orientación N-S (GEOCONSA, 1999).

Muchos de los sistemas de drenaje superficial están sobre un sistema de fallas, como los ríos Guadrón, Pinula y quebrada del Frutal. En lo que corresponde al río Villalobos, está ubicado sobre una depresión (graben) definida por fallas verticales. Con esto se puede indicar que existe un control estructural geológico sobre las principales corrientes superficiales, por consiguiente existe un control estructural sobre el flujo de aguas subterráneas. Además, se nota un sistema de fallas y fracturas pequeñas con orientación NW-SE y E-W en los alrededores de Ojo de Agua, que corresponden con pequeños bloques basculados de norte a sur. Por esto, se encuentran mayores espesores de sedimentos en los pozos hacia el sur de Ojo de Agua (Figs 3 y 4). 


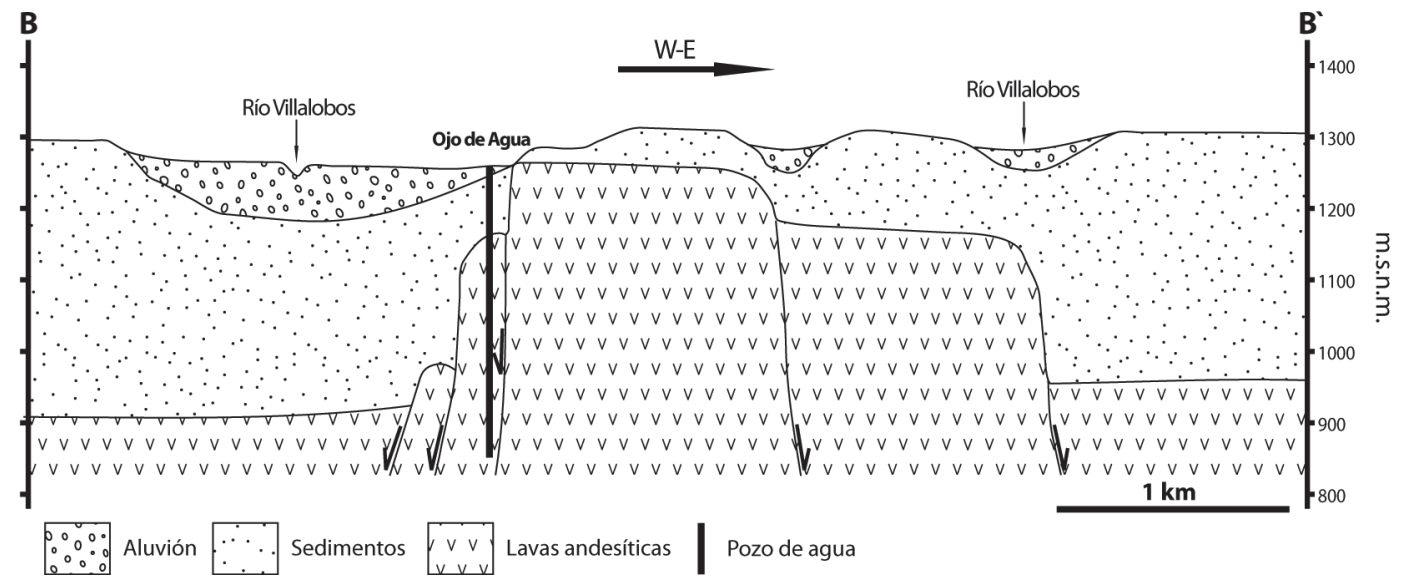

Fig. 4: Perfil geológico B-B'

\section{HIDROGEOLOGÍA}

\section{Acuíferos del área de estudio}

Hidrogeológicamente el área de estudio se encuentra conformada por dos acuíferos importantes: Acuífero Superior y Acuífero Inferior (Jiménez, 1990). Estos acuíferos se delimitan de mejor manera en la parte sur del área, donde se localizan los depósitos aluviales de los ríos Villalobos y Pinula, los sedimentos fluvio-lacustres y las lavas andesíticas en la base (ver figura 5).

\section{a) Acuifero superior}

El Acuífero Superior es de tipo libre o freático y está constituido esencialmente por sedimentos aluviales depositados en las partes media e inferior de los valles de los ríos Villalobos y Pinula que tienen una extensión de $60 \mathrm{~km}^{2}$ y un espesor de 10 a $30 \mathrm{~m}$. Existen localmente intercalaciones de sedimentos fluvio-lacustres, paleosuelos y pómez que presentan espesores de más de $300 \mathrm{~m}$, actuando en algunas partes como acuicludos, debido a su granulometría arcillo-limosa. Se incluye dentro de este acuífero superior los depósitos cuaternarios de piroclastos pomáceos compactos hasta sueltos, mal clasificados y mal estratificados. Abajo de estos sedimentos se encuentran las lavas terciarias que forman la base del valle sur de ciudad Guatemala con una extensión mayor de $200 \mathrm{~km}^{2}$ (Herrera, 2002).

\section{b) Acuifero inferior}

El Acuífero Inferior es de tipo confinado y está constituido principalmente por lavas andesíticas, las cuales subyacen al acuífero superior. Las lavas por sus características de alta permeabilidad (porosidad secundaria), fracturación, extensión y espesor (mayor de $400 \mathrm{~m}$ ), constituyen el principal acuífero del área (GEOCONSA, 1999). En algunos pozos de Ojo de Agua, el espesor de lavas es superior a $200 \mathrm{~m}$. Se asume que los pozos del Diamante están captando el acuífero superior, repercutiendo esto en la entrada a los pozos de una gran cantidad de sedimentos, mientras que los pozos del sector Ojo de Agua, la explotación se realiza en el acuífero inferior de lavas.

\section{Características hidrogeológicas}

El acuífero superior tiene diferentes tamaños granulométricos, siendo el material de relleno de diferentes dimensiones que varían desde bloques y gravas de rocas volcánicas hasta arenas de pómez, limos y arcillas, presentando una permeabilidad primaria. El agua subterránea se encuentra en su mayor parte bajo condiciones libres y semiconfinadas, esto último, por existir intercalaciones de 
sedimentos lacustres y paleosuelos. Los valores de transmisividad para los piroclastos varían de 50 a $750 \mathrm{~m}^{2} /$ día, mientras que los sedimentos aluviales del río Villalobos tienen una transmisividad entre 150 a $2000 \mathrm{~m}^{2} /$ día (Yol, 2002). Los valores de coeficiente de almacenamiento asignado para los depósitos piroclásticos es 0,09 y para los depósitos aluviales de 0,20 (Velásquez, 1998).

El acuífero inferior presenta una permeabilidad secundaria, encontrándose bajo condiciones de confinamiento (López, 1989). Esto por la situación de que sobre el acuífero se encuentran los piroclastos compactos y sedimentos finos, los cuales son prácticamente impermeables. La transmisividad varía entre 500 y $5000 \mathrm{~m}^{2} /$ día, asignando un valor de $8 \times 10^{-3}$ al coeficiente de almacenamiento para rocas volcánicas fracturadas del valle de Guatemala (Herrera, 2002).

Por pruebas de bombeo efectuadas por EMPAGUA y empresas consultoras, los valores de transmisividad oscilan entre 1600 a $9500 \mathrm{~m}^{2} /$ día en los pozos de Ojo de Agua y entre 22 a $1300 \mathrm{~m}^{2} /$ día en el sector de El Diamante (Cuadro 1). Los datos de los niveles estáticos de los pozos, los caudales y las transmisividades son del año cuando se perforaron los pozos, presentándose actualmente diferentes valores de estas características en varios pozos.

Es claro que la explotación de agua subterránea con pozos debe dirigirse a captar el acuífero inferior en lavas para obtener mayores producciones de agua. Siendo necesario efectuar pruebas de bombeo de pozos con piezómetros colocados a cortas distancias $(10$ a $30 \mathrm{~m})$ y distintas profundidades para calcular de forma precisa los parámetros hidrogeológicos del área y de esta forma definir a detalle los tipos de acuíferos.

\section{Niveles y movimiento de las aguas subterráneas}

Al momento del inicio de explotación en el sector Ojo de Agua (1976) los niveles estáticos estaban muy cercanos a la superficie del terreno. En el caso del pozo Ojo de Agua 2, el nivel se encontraba a una cota superior a la boca del pozo o sea como surgente.

Con el objeto de conocer la variación de los niveles estáticos del agua subterránea en el área de estudio, se compararon los niveles medidos en ocho pozos al momento de la terminación de los mismos $(1968,1970$ y 1976) hasta la última medición reportada en mayo de 1996 para los mismos ocho pozos, ya que no todos los pozos de Ojo de Agua y El Diamante han sido medidos, como se presenta en el cuadro 2 .

Se observa que los niveles estáticos de los pozos descendieron en promedio de 9 metros en el sector Ojo de Agua. Mientras que en el sector de El Diamante descendieron un promedio de 25 m en más de 30 años. Sólo el pozo El Diamante 7 (que se encuentra más próximo al río Villalobos) ha tenido un menor descenso del nivel estático.

De acuerdo a los datos de niveles estáticos de los pozos de Ojo de Agua y otros pozos de los alrededores medidos en el año 1996, y por medio de la interpolación visual de mapas y secciones, se definieron las isopiezas y el movimiento del agua subterránea para el acuífero inferior (figura 6). Se observa que la dirección de flujo de las aguas subterráneas en el área, es de norte a sur y noreste a suroeste con un control estructural de las fallas de la quebrada del Frutal y río Pinula, que definen un pequeño horst entre los ríos Villalobos y Pinula, separando dos flujos de aguas subterráneas del acuífero sur del valle.

Los principales flujos de aguas subterráneas provienen del oeste y tienen su recarga en los alrededores de Mixco (López, 1989) y el otro flujo de aguas subterráneas (menor que el primero), se recarga en las partes montañosas de Santa Catarina Pinula (GEOCONSA, 1999), siendo la causa que los dos flujos están separados tectónicamente y se unen en el sector de Ojo de Agua.

\section{DESARROLLO DE LAS AGUAS SUBTERRÁNEAS}

\section{Explotación actual de las aguas subterráneas}

Los sistemas Ojo de Agua y Diamante, tienen como fuente principal el agua proveniente de los acuíferos. Estos son explotados por medio de pozos con bomba de diferentes diámetros y profundidades, como se describe a continuación en forma resumida. 


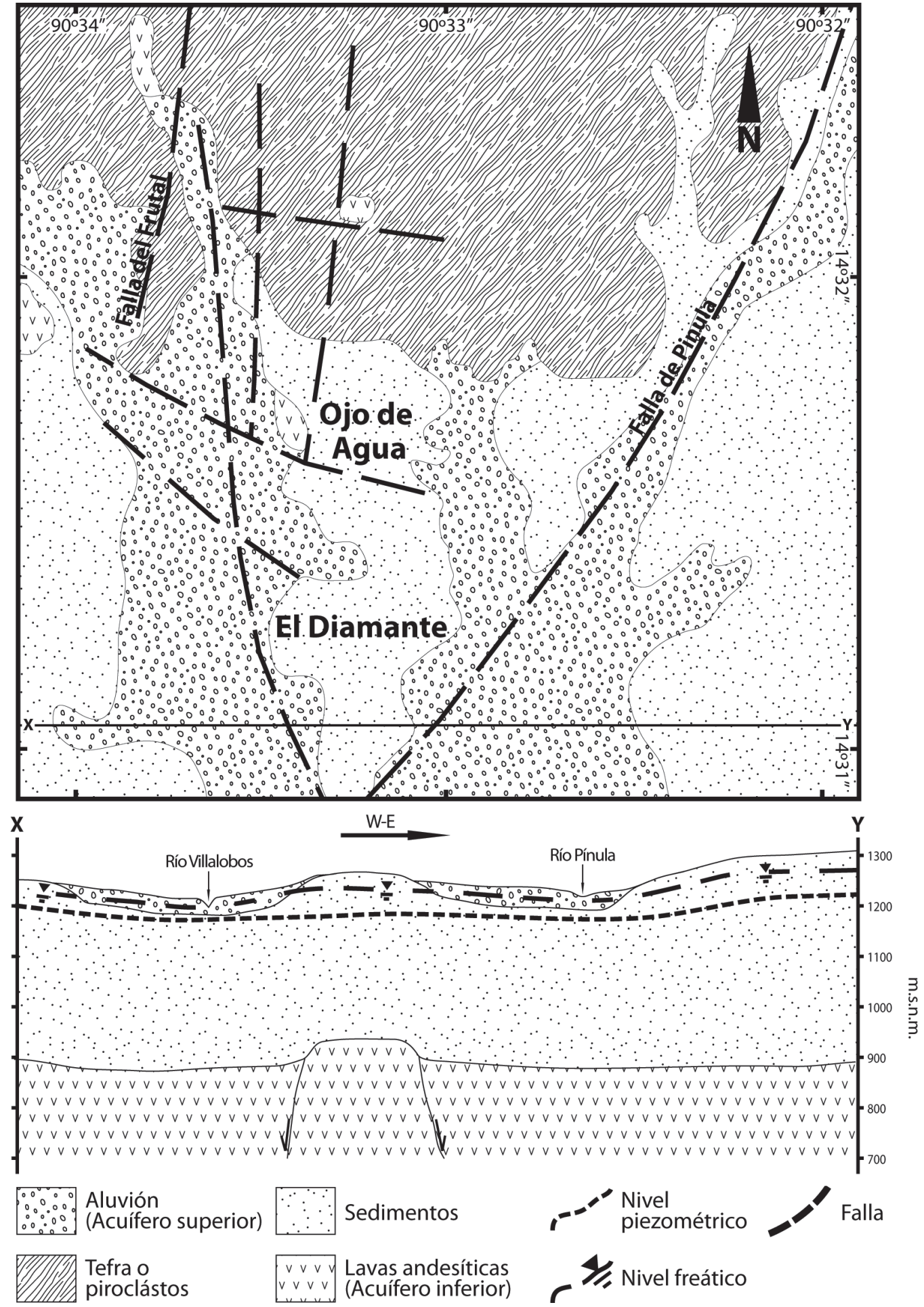

Fig. 5: Perfil hidrogeológico del área 
Cuadro 1

Características de algunos pozos existentes en los sectores Ojo de Agua y Diamante

\begin{tabular}{|c|c|c|c|c|c|c|c|c|}
\hline Pozo & $\begin{array}{c}\text { Año } \\
\text { const. }\end{array}$ & $\begin{array}{l}\text { Nivel estático } \\
\text { (m.b.n.s.) }\end{array}$ & $\begin{array}{c}\text { Altitud } \\
\text { (m.s.n.m.) }\end{array}$ & $\begin{array}{l}\text { Prof. } \\
\text { (m) }\end{array}$ & $\begin{array}{c}\text { Diámetro de } \\
\text { encamisado (pulg) }\end{array}$ & $\begin{array}{c}\text { Caudal } \\
(1 / \mathrm{s})\end{array}$ & $\begin{array}{c}\mathrm{T} \\
\left(\mathrm{m}^{2} / \text { día }\right)\end{array}$ & $\begin{array}{l}\text { Estado } \\
\text { actual }\end{array}$ \\
\hline Ojo de Agua 1 & 1976 & 0,90 & 1247,9 & 280,50 & 12 a 8 & 134 & 5530 & E.P. \\
\hline Ojo de Agua 2 & 1976 & artesiano & 1253,4 & 274,40 & 12 a 8 & 138 & 9504 & E.P. \\
\hline Ojo de Agua 3 & 1987 & 0,60 & 1252,3 & 274,40 & 20 a 12 & 144 & 4320 & E.P. \\
\hline Ojo de Agua 4 & 1987 & 3,00 & 1255,0 & 365,80 & $20-12$ a 8 & 207 & 1642 & E.P. \\
\hline Ojo de Agua 5 & 1990 & 6,00 & 1258,2 & 350,60 & 12 & 174 & --- & E.P. \\
\hline Ojo de Agua 6 & 1991 & 4,50 & 1258,9 & 350,00 & 12 & 207 & --- & E.P. \\
\hline Ojo de Agua 7 & 2002 & 9,50 & 1259,2 & 330,00 & 16 & 218 & --- & E.P. \\
\hline Ojo de Agua 8 & 2002 & 10,00 & 1259,1 & 330,00 & 16 & 221 & --- & E.P. \\
\hline Ojo de Agua 9 & 2003 & 13,00 & 1258,7 & 350,00 & 16 & 232 & 5335 & E.P. \\
\hline Piezómetro & 1975 & 7,23 & 1255,0 & 80,38 & 12 & --- & --- & Observación \\
\hline Diamante 1 & 1968 & 9,10 & 1252,6 & 158,50 & 12 & 34 & 121 & E.P. \\
\hline Diamante 2 & 1968 & 7,60 & 1247,9 & 142,40 & 16 a 12 & 13 & 22 & $?$ \\
\hline Diamante 3 & 1969 & 7,60 & 1237,1 & 128,05 & 16 a 12 & 50 & 1296 & $?$ \\
\hline Diamante 4 & 1970 & 18,30 & 1248,0 & 262,20 & 16 & --- & -- & A. \\
\hline Diamante 5 & 1970 & 7,60 & 1250,6 & 312,80 & 16 & 51 & 73 & E.P. \\
\hline Diamante 6 & 1973 & 33,50 & 1245,0 & 311,00 & 16 & --- & --- & A. \\
\hline Diamante 7 & 1976 & 2,20 & 1247,8 & 305,00 & 12 a 8 & 39 & 233 & E.P. \\
\hline Diamante 8 & 1976 & 19,50 & 1249,0 & 274,40 & 12 a 8 & --- & 164 & A. \\
\hline Diamante 9 & 1989 & 16,97 & 1247,7 & 311,00 & 12 & --- & --- & A. \\
\hline
\end{tabular}

T: Transmisividad, D.e.: Diámetro de encamisado

E.P.: En producción, A: Abandonado

Fuente: Archivo de pozos. EMPAGUA

\section{a) Sector Ojo de Agua}

En este sector se encuentran ubicados nueve pozos mecánicos denominados Ojo de Agua (OA), un tanque de compensación y la estación de bombeo. En este campo se extrae un caudal de $1675 \mathrm{l} / \mathrm{s}$. Las producciones parciales en 1/s son: OA-1 con 134, OA-2 con 138, OA-3 con 144, OA- 4 con 207, OA-5 con 174, OA-6 con 207, OA-7 con 218, OA-8 con 221, y OA-9 con 232.

\section{b) Sector El Diamante:}

En este sector se encuentran ubicados los pozos Diamante 1, 2, 3, 4, 7, 8 y 9, así como las tuberías de conducción que envían el caudal explotado en esta zona hacia el tanque de compensación del sector de Ojo de Agua.

\section{c) Sector Diamante Anexo}

En este sector se encuentran ubicados los pozos denominados Diamante 5 y Diamante 6, y sus caudales son transportados hacia el tanque de compensación en el sector Ojo de Agua. Actualmente ya no se explotan todos los pozos que se encuentran ubicados en el sector Diamante, ya que han tenido que ser abandonados algunos por problemas de producción de sedimentos finos (limo y arena), colapsos en la tubería del pozo o por aparición de contaminantes químicos. Han quedado fuera de servicio los pozos Diamantes 
Variación de los niveles estáticos de agua de los pozos en m.s.n.m.

\begin{tabular}{|c|c|c|c|c|c|}
\hline \multirow[t]{2}{*}{ Pozo } & \multicolumn{2}{|c|}{$\begin{array}{l}\text { Primer año de medición } \\
\text { del nivel estático }\end{array}$} & \multicolumn{2}{|c|}{$\begin{array}{l}\text { Último año de medición } \\
\text { del nivel estático }\end{array}$} & \multirow[t]{2}{*}{$\begin{array}{l}\text { Variación del } \\
\text { nivel estático } \\
\text { (m) }\end{array}$} \\
\hline & Año & m.s.n.m & Año & m.s.n.m & \\
\hline Ojo de Agua 1 & 1976 & 1247,0 & 1996 & 1240,5 & $-6,5$ \\
\hline Ojo de Agua 2 & 1976 & 1254,0 & 1996 & 1247,0 & $-7,0$ \\
\hline Ojo de Agua 3 & 1987 & 1251,7 & 1996 & 1238,5 & $-13,2$ \\
\hline Ojo de Agua 4 & 1987 & 1252,0 & 1996 & 1242,0 & $-10,0$ \\
\hline Diamante 1 & 1968 & 1243,5 & 1996 & 1212,5 & $-31,0$ \\
\hline Diamante 3 & 1989 & 1229,5 & 1996 & 1207,0 & $-22,5$ \\
\hline Diamante 5 & 1970 & 1243,0 & 1996 & 1223,0 & $-20,0$ \\
\hline Diamante 7 & 1976 & 1245,6 & 1996 & 1236,5 & $-9,1$ \\
\hline
\end{tabular}

Fuente: EMPAGUA, 1996

4, 6, 8 y 9, los cuales son utilizados como piezómetros de observación. La producción total de los pozos Diamante 1, 2, 3, 5 y 7 es de 187 1/s.

\section{Potencial de los recursos hídricos subterráneos}

Para determinar si existe sobre-explotación del acuífero, se compararon los volúmenes totales de la recarga y de la extracción de las aguas subterráneas de forma temporal (anual). Cualquier explotación continua de aguas que excede la recarga natural, está acompañada por un descenso continuo de los niveles. Tal descenso, causará una disminución continua en los caudales de los pozos y en las salidas del acuífero. Por estos hechos, es preferible que el ritmo de la explotación de las aguas sea menor que la recarga natural.

Si la construcción de los pozos está bien planificada, tomando en cuenta los descensos pronosticados de los niveles, los caudales de los pozos pueden mantenerse sin disminuirse.

De acuerdo al "Estudio de los Recursos de Aguas Subterráneas para el abastecimiento a la Ciudad de Guatemala" (Tahal Consulting Engineers Ltd., 1990), el bombeo neto del sistema acuífero del valle de Guatemala, es menor que la recarga natural, por lo que se puede aumentar el bombeo dentro del valle, con un descenso permisible máximo del nivel del agua de $35 \mathrm{~m}$. Ya que se asume que descensos más grandes pueden disminuir considerablemente el espesor del acuífero, disminuyendo así su transmisividad y afectando adversamente los caudales de los pozos, que se requiere en este caso de profundizarlos.

Velásquez (1998), menciona que la explotación del agua subterránea en los sectores Ojo de Agua y El Diamante ha mostrado que existe un potencial de diferentes características y magnitudes, pero se considera probable obtener caudales adicionales que permitan ampliar la producción de agua de EMPAGUA. Además, se refiere a que los descensos de los niveles del agua subterránea en ambos sectores no significan que los acuíferos se estén agotando, ya que por una parte estos descensos varían entre 10 a $20 \mathrm{~m}$, mientras que las profundidades de explotación son menores de 300 metros y la recuperación de los pozos es relativamente rápida. Velásquez (1998) concluye que explotar medio metro cúbico por segundo adicional en ambos sectores es factible, sin embargo las características hidrogeológicas en ambos sectores difieren sensiblemente y el potencial en cada uno de ellos es diferente. 


\section{Áreas propuestas para nuevos pozos de agua}

A sabiendas de que las producciones de los pozos perforados en el sector Ojo de Agua son mucho mayores que los obtenidos en El Diamante, y por los resultados hidrogeológicos y geofísicos (GEOCONSA, 1999), es recomendable establecer nuevos pozos en el acuífero inferior de lavas a más de 300 metros de profundidad.

Priorizando las 2 áreas para la exploración con la perforación de pozos, se tiene:

1) Al sur de Ojo de Agua, entre las coordenadas $14^{\circ} 31^{\prime} 37^{\prime \prime} \mathrm{N}-90^{\circ} 33^{\prime} 31^{\prime \prime} \mathrm{O}$.

2) $\mathrm{Al}$ este de Ojo de Agua, entre las coordenadas $14^{\circ} 31^{\prime} 39^{\prime \prime} \mathrm{N}-90^{\circ} 33^{\prime} 01^{\prime \prime} \mathrm{O}$.

La primera área es propuesta (al sur de Ojo de Agua), por los datos geofísicos y por la litología del pozo Ojo de Agua 1 y Diamante 9, que muestran que la profundidad de las lavas andesíticas es alrededor de los $100 \mathrm{~m}$ y que el espesor de estas rocas volcánicas es mayor de los $200 \mathrm{~m}$. De aquí se interpreta, que se podría captar el acuífero inferior, el cual presenta una mayor producción de agua. El área 2 por fotointerpretación y geofísica (GEOCONSA, 1999), muestra un alto grado de fracturación y una posible zona de lavas cerca de la superficie, que por falta de información de pozos debe ser comprobada con alguna perforación de exploración.

Diferentes estudios de López (989), Jiménez (1990), BRGM-INSIVUMEH (1997), Velásquez (1998) y GEOCONSA (1999) en el valle sur de ciudad Guatemala, definen claramente que la dirección de flujo tiene una confluencia en los alrededores del sector de Ojo de Agua, dando un alto grado de certeza de que en este lugar se tiene un gran flujo de agua subterránea.

En éstas dos áreas, la explotación de medio metro cúbico por segundo, se puede lograr por medio de la perforación de por lo menos 3 pozos, a profundidades de $400 \mathrm{~m}$, en los cuales se podría obtener en cada uno de ellos un caudal mínimo de $170 \mathrm{l} / \mathrm{s}$, con lo que se alcanzaría una producción conjunta de 510 1/s.

Es importante de tomar en cuenta que las áreas del sector El Diamante y Diamante Anexo,

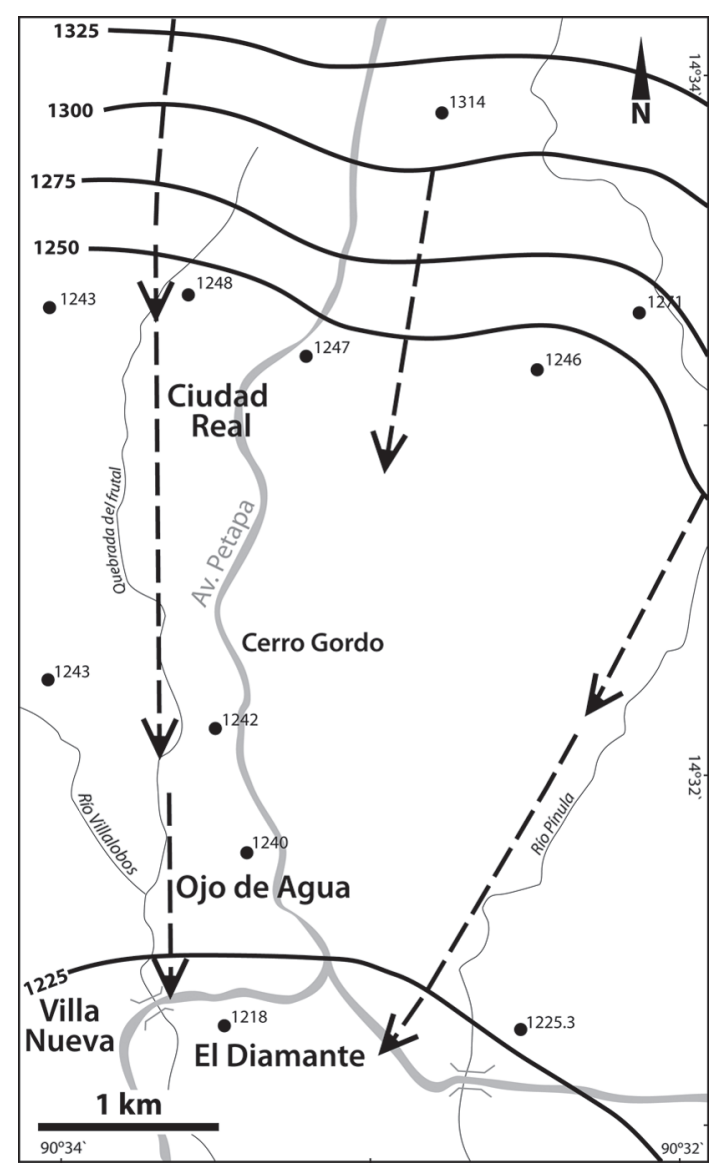

Fig. 6: Mapa de flujo del agua subterránea

presentan un gran espesor de sedimentos, principalmente al este y al sur, como lo demuestran los perfiles litológicos de los pozos, siendo una zona de baja producción, si se compara con el sector de Ojo de Agua. Por lo anterior, estas áreas no son recomendables de explorar, ya que tienen el agravante de contener muchos sedimentos finos que incluso han causado problemas serios en los pozos de los sectores de El Diamante.

\section{CONCLUSIONES}

En el área del valle sur de ciudad Guatemala se identifican dos acuíferos: uno superior de origen 
aluvial y el otro inferior formado por lavas volcánicas fracturadas que se extiende a más de $400 \mathrm{~m}$ de profundidad con un espesor superior a los $200 \mathrm{~m}$.

El acuífero en lavas volcánicas tiene mejores características hidrogeológicas (transmisividades del orden de miles de $\mathrm{m}^{2} /$ día), siendo mejor captar éste y aislar el acuífero superior.

El acuífero explotado en el sector de Ojo de Agua produce un total de 1675 1/s (con 9 pozos) y presenta un descenso en los niveles de agua subterránea que alcanzan valores de hasta $13 \mathrm{~m}$ en más de 20 años de explotación.

Los estudios hidrogeológicos realizados en el valle sur de Guatemala, muestran que el potencial de agua subterránea en esta área, permite extraer caudales adicionales de agua subterránea en zonas cercanas al sector de Ojo de Agua.

Este estudio sirvió para priorizar dos áreas de interés hidrogeológico, pero no se pueden garantizar los caudales a producir hasta realizar las pruebas de eficiencia de pozos. Ya que los caudales estarán en función del buen diseño y el mejor acabado de los pozos.

\section{RECOMENDACIONES}

Se recomiendan dos áreas prioritarias para la perforación de pozos de agua, donde la profundidad de perforación debe realizarse entre los 400 a $600 \mathrm{~m}$. Siendo recomendable realizar primeramente una perforación de exploración con un pozo de diámetro reducido (el cual puede servir posteriormente como piezómetro), obteniendo el perfil litológico real del área.

Perforar los pozos productores en diámetros amplios (tubería no menor de 12 pulg de diámetro) y usar tubos de rejillas hechos con medios mecánicos y no con acetileno

En las áreas donde los pozos atraviesen las lavas, debe tratarse de aislar completamente la parte superior de piroclastos y aluvión, para evitar la entrada de sedimentos finos al pozo y la posible contaminación proveniente del acuífero aluvial.

Al terminar la perforación, hay que realizar en cada pozo productor una prueba de bombeo con al menos un piezómetro instalado cerca de éste (10 a $30 \mathrm{~m}$ de distancia), para determinar los parámetros hidrogeológicos y la distancia recomendable para que no exista interferencia entre los pozos. Además, realizar en todos los nuevos pozos, una prueba de bombeo escalonada para determinar la eficiencia del pozo.

\section{AGRADECIMIENTOS}

Se agradece a los Ingenieros Carlos Tobar y Octavio Leiva por la ayuda prestada para la realización de este estudio. Al Ing. Julio Taracena se le agradece y reconoce el apoyo brindado en la digitalización y elaboración de los mapas y figuras de esta investigación. Un agradecimiento especial a los funcionarios de EMPAGUA por proporcionar información hidrogeológica del área de estudio. Se agradece los comentarios de Percy Denyer, así como, las revisiones y sugerencias de Gerardo Soto y Marco Barahona.

\section{REFERENCIAS}

HERRERA, I., 1998: Reconocimiento hidrogeológico de la cuenca del río Itzapa, Chimaltenango, Guatemala.- 104 págs. Univ. de Costa Rica, San José [Tesis M.Sc.].

HERRERA, I., 2002: Hidrogeología Práctica [1 $1^{\mathrm{a}}$ ed.].- 345 págs. Univ. de San Carlos de Guatemala, Guatemala.

BRGM-INSIVUMEH，1997: Modelización del transporte de agua y solutos en los estratos volcánicos y sedimentarios en la Ciudad de Guatemala.- 144 págs. Proyecto de Cooperación Internacional FrancoGuatemalteco, Guatemala [Inf. interno].

GEOCONSA, 1999: Informe hidrogeológico de "Ojo de Agua" y alrededores del sector sur de ciudad Guatemala.- 24 págs. EMPAGUA, Guatemala [Inf. técnico].

INSIVUMEH-IGN-ONU, 1978: Estudio de las Aguas Subterráneas en el Valle de 
Guatemala.- 303 págs. Ministerio de Comunicaciones y Obras Públicas, Guatemala.

\section{INSTITUTO NACIONAL DE ESTADISTICA,} 2002: Censos Nacionales XI de Población y VI de Habitación.- 272 págs. Gobierno de Guatemala, Guatemala.

JIMENEZ, M., 1990: Estudio Hidrogeológico de los acuíferos del sector de Ojo de Agua El Diamante Zona 12, Guatemala.- 141 págs. Univ. de San Carlos de Guatemala, Guatemala [Tesis M.Sc.].

KOCH, A. \& McLEAN, H., 1975: Pleistocene tephra and ash flow deposits in the volcanic highlands of Guatemala.- Geol. Soc. Am. Bull. 86: 529-541.

KOCH, A. \& McLEAN, H., 1977: Mapa Geológico de Ciudad Guatemala.- Escala 1:50 000, IGN, Guatemala.
LOPEZ S., 1989: Evaluación Hidrogeológica del Ojo de Agua y El Diamante.- 129 págs. Univ. de San Carlos de Guatemala, Guatemala [Tesis Lic.].

TAHAL CONSULTING ENGINEERS LTD., 1990: Estudio de Recursos de Agua. Tomo I. Los Recursos de agua subterránea para el abastecimiento a la Ciudad de Guatemala. Proyecto de Rehabilitación del abastecimiento de agua a la ciudad de Guatemala.189 págs. EMPAGUA [Inf. interno].

VELÁSQUEZ E., 1998: Estudio Hidrogeológico de los acuíferos aluviales del río Villalobos.21 págs. EMPAGUA, Guatemala [Inf. técnico].

YOL, V., 2002: Evaluación y propuesta de manejo y uso sostenible del recurso hídrico de la finca Sabana Grande, El Rodeo, Escuintla.- 107 págs. Univ. de San Carlos de Guatemala, Guatemala [Tesis Lic.]. 
